\title{
NEUROSIS Y GASTRALGIA SEGÚN JUAN BTA. PESET Y VIDAL (1869)
}

\author{
Mariano Peset Mancebo \\ Universidad de Valencia - Instituto de Estudios Documentales e \\ Históricos sobre la Ciencia. C.S.I.C. Valencia (España)
}

\section{RESUMEN}

El artículo consta de dos partes. La primera es un resumen del tratado en donde se apuntan las ideas más importantes sobre la enfermedad analizada por Peset y Vidal. En una segunda parte, se realiza un análisis comparativo del texto, utilizando libros de la época y materia tratados por el autor, así como diccionarios médicos. En definitiva, el autor presenta la "gastralgia" como una neurosis digestiva.

\section{SUMMARY}

The article consist of two parts. The first is a summary of the treaty in which the main ideas about diseases analysed by the doctor Peset and Vidal are presented. The second part is a comparative analysis of the text for which the author has consulted many books and other material of those days, as well as medical dictionaries. As a result of it, the author presents the "gastralgia" as a digestive neurosis.

Peset y Vidal publica en 1869 su Historia de las neurosis llamadas digestivas y en especial de la gastralgia. Es un librito o folleto de ciento cuarenta y nueve páginas que nos ha servido para adentrarnos en su pensamiento médico. A través de él, se puede conocer cómo concebía la enfermedad y cuál era el nivel de su información médica.

Procederé, en primer lugar, a describir el contenido de estas páginas para recoger sus ideas de forma sencilla y ordenada. De este modo se puede apreciar su valoración de la enfermedad y sus diversos aspectos, que organiza con precisión. A continuación, me permitiré un comentario o valoración de sus ideas, para enmarcarlas en la medicina de su tiempo. 


\section{NEUROSIS DIGESTIVAS: UN TRATADO SINTETIZADOR}

Según Peset, no hay que denominar nerviosa toda enfermedad que presente síntomas nerviosos. Unicamente se consideran tales aquéllas cuya lesión desconocida reside en el sistema nervioso y no tengan otra causa orgánica. La autopsia demuestra que muchas enfermedades consideradas nerviosas, eran debidas a un vicio orgánico que afectaba a alguna parte del cuerpo y no habían sido vistas en vida.

La ausencia de lesión es una prueba de que este tipo de enfermedades no admite, como causa, lesión alguna anatómica apreciable. Secuela de lo dicho es la poca fijeza, la volubilidad de todos los síntomas que, presentándose instantáneamente y de manera imprevista, desaparecen de repente.

Por consideraciones etiológicas las neurosis se dividen en primitivas o idiopáticas y secundarias o sintomáticas. Las primeras provienen inmediatamente de las causas morbosas y su acción sobre el sistema nervioso; las segundas resultan de otra enfermedad. En realidad no hay más neurosis que las esenciales y únicamente lo son las primeras o idiopáticas, considerando los otros grupos como síntomas o elementos constitutivos de enfermedades dadas.

Ultimamente las neurosis, bien sean de la vida animal o de la vida orgánica, se subdividen según los aparatos orgánicos que comprenden: clasificando así las neurosis de la vida animal en el cerebro, sentidos externos y demás órganos que le corresponden; e igualmente las de vida orgánica o nutritiva, las digestivas, respiratorias, circulatorias y otras referentes a los diversos órganos.

A continuación entra en la consideración de las digestivas. Las gastralgias o neurosis digestivas son neurosis de sensación y movimiento, que tienen su asiento en el sistema nervioso de los diferentes órganos que componen el aparato digestivo, cuyos caracteres generales y trastornos de sus funciones son el dolor y la convulsión. Un signo importante que acompaña siempre estas neurosis, y que sirve para su diagnóstico, es la contracción convulsiva o el espasmo de la túnica muscular.

\section{DE LA GASTRALGIA}

Etimología, sinonimia y definición. La gastralgia es una neurosis menos importante por su violencia o peligro inminentes. Es una enfermedad crónica y aunque desaparece o se oscurece, el individuo queda expuesto a nuevos ataques, tan intensos como el primero. Es una afección de esencia desconocida y cuya curación es eventual, porque se desconoce su tratamiento específico. Es una enfermedad antigua que se conocía ya en la época de Hipócrates. Para Peset es mejor el nombre de Gastrosis nerviosa o dinámica o enfermedad del estómago por vicio en su inervación. También la denominaría dispepsalgia que expresa bien sus principales caracteres. Se ha cono- 
cido también por el nombre de cardialgia, así llamada por la abertura superior del estómago, porque en ella se creyó residían el dolor y la enfermedad. Algunos autores más antiguos la denominan dolor del corazón.

Etiología, predisposición, causas determinantes. Existe una constitución especial, enfermiza, con cierta disposición orgánica muy análoga al temperamento linfático nervioso, que puede denominarse gastrálgica. Esta enfermedad aparece en los temperamentos nerviosos, sobre todo si disfrutan de una idiosincrasia gastrohepática. El individuo que la padece tiene una constitución delgada y seca, rostro pálido y ligeramente contraído, con aspecto malsano.

Existen muchos casos de gastralgia de transmisión de padres a hijos; por tanto es fácil admitir la herencia de esta afección, «o hablando con más propiedad, una conformación orgánica particular heredada».

También - en especial en la dispéptica — se da la excesiva secreción y excreción de los humores recrementicios de la economía. Y se desarrollan gastralgias en los estados particulares fisiológicos como la menstruación y el embarazo, así como la leucorrea, herpes, gota y reumatismo. Existen otras causas llamadas indirectas, que aunque muy numerosas, provienen de tres fuentes principales y son: las pasiones tristes y concentradas, el exceso de los placeres de Venus y los excesivos trabajos intelectuales sin el suficiente descanso.

Anatomía patológica. La mayoría de autores que comprenden bien lo que significa la palabra gastralgia pasan por alto el estudio de sus lesiones anatómico-patológicas. No puede ser de otra forma puesto que hay una ausencia de lesión en el estómago y demás vísceras. La falta de lesiones caracteriza esta neurosis ${ }^{1}$.

Sintomatología. Los síntomas de la gastralgia corresponden a tres órdenes. Los primeros locales o sea las alteraciones funcionales del estómago; los segundos vienen dados por las fuertes simpatías de este órgano con los demás de la economía; los terceros son relativos al estado de nutrición general, consecuencia del desarreglo de la digestión. Entre los síntomas se dan: calenturas, que cesan después de la comida o de haber vomitado; pirosis o sensación de ardor en la faringe y esófago; eructos molestos y vómitos; bulimia; dispepsia o dificultad para las digestiones. La sensación molesta o dolorosa en la región epigástrica desarrollada por la enfermedad, —el dolor- es uno de los síntomas constantes, aunque no indispensable. Es un dolor vago y sordo que pasará a ser vivo y atroz y en ocasiones insoportable. Suele ser continuo en los ataques gastrálgicos, con intervalos regulares de aumento y disminución. Periódico, pero en las remisiones subsiste leve o, al menos, una sensación de angustia inexplicable.

1 PESET ReIG, J. L. (1993), Las heridas de ciencia, Junta de Castilla y León, Valladolid. La anatomía patológica de los alienados, no considerados éstos como neuróticos, también ha permanecido muda sobre la sede de la locura. Esquirol dice que los cuadros psíquicos son complejos y, muchas veces, no presentan traducción anatomopatológica, pp. 174-175 y ss. 
La digestión ordinariamente es penosa (dispepsia) y se verifica mal. Al ser lenta produce una sensación de peso, tirantez incómoda, tensión en el epigastrio.

Marcha y duración. Terminación. En el desarrollo de la gastralgia se puede encontrar tres períodos, enlazándose en cada uno sus síntomas. Estos períodos son: invasión, declaración y declinación. Lo normal es que llegue precedida por unos cuantos días con síntomas dispépsicos o dolores vagos. Sus ataques no tienen plazo fijo para su presentación e intensidad. Los ataques son cortos en la forma dolorosa y tienen mayor duración en las formas hipocondríaca y dispéptica. La duración de la enfermedad es varia, aunque se la ha observado de treinta años, lo normal es que dure de dos meses a doce años. Igual ocurre si el enfermo no guarda un régimen higiénico severo. Tiene corta duración la que algunos autores llaman gastralgia aguda, que consiste en la manifestación de síntomas propios de esta neurosis.

La neurosis digestiva nunca termina por la muerte y su tendencia natural es a la curación. A veces ocurren degeneraciones en el estómago y se desarrollan en dicha víscera lesiones orgánicas. Se observan cánceres al hacer la autopsia en personas que anteriormente habían tenido la neurosis que nos ocupa.

Formas de la gastralgia. La gastralgia presenta varias formas, las cuales, atendiendo a su síndrome, se reducen fácilmente a tres: la dolorosa, la dispépsica y la hipocondríaca. Los síntomas, aunque pueden aparecer aisladamente, lo normal es que vengan todos reunidos, aunque el autor considera diferentes formas según predomine uno u otro. En la forma «dolorosa» sobresale un síntoma culminante que es el dolor en epigastrio. Este dolor puede ser mediano, obtuso, gravativo, distensivo y atroz. Unas veces se presenta dilacerante, constrictivo y convulsivo, otras quemante o glacial. Generalmente el dolor está limitado a los órganos de la región, pero a veces se extiende a las partes inferiores del vientre o al dorso, esternón, escápulas y costados.

La forma «dispépsica» es, por lo general, más benigna, pero de más larga duración. Presenta atonía, espasmo, acedías, flatulencias, opresión y ansiedad epigástrica. Los síntomas son locales o concretados al estómago, generales y de toda la economía.

Coloca la «hipocondría» entre las neurosis. Se atribuye a un desorden particular del sistema nervioso, que determina el estado triste habitual, los temores, el desaliento moral y desarreglos en la digestión. Se diferencia la hipocondría esencial de la gastralgia, en que en ésta se observan padecimientos orgánicos; mientras, en la hipocondría esencial son morales, pero esto no quiere decir que no sea real, sino que la fantasía la agrava y la sagacidad del espíritu duplica sus dolores y padecimientos. Por ello aparece más en gente desocupada que entre los trabajadores, ya que la fatiga embota la sensibilidad y provoca sueño. Opina Trousseau y otros prácticos que todas las enfermedades nerviosas del hombre puedan referirse a un mismo grado patológico, y deben agruparse alrededor de la forma hipocondríaca. 
Las complicaciones más frecuentes en la gastralgia, son las demás enfermedades de su misma naturaleza, o sea las nerviosas. La enteralgia o neurosis intestinal aparece acompañando a la gastralgia y da lugar a que muchos autores la denominen gastro-enteralgia. Pero se observan casos de gastralgia y enteralgia separadas, por lo que no parece correcto reunirlas. Además a veces aparece la enteralgia después de un ataque dispéptico. Otras son el histerismo, la neurosis hepática, la neuralgia del cordón espermático.

Su naturaleza es desconocida, aun en su esencia íntima. Sin embargo hay pocas enfermedades que hayan sido objeto de tanta investigación. La anatomía patológica, «antorcha de los conocimientos modernos», no ha contribuido a ilustrar su origen, pero ha reducido a polvo las hipótesis más o menos deslumbrantes.

Con notable erudición acomete la historia de esta enfermedad. Para Hipócrates era debida a un predominio y desenfreno de la bilis amarilla, mientras Galeno la achaca a un efecto de las crudezas del estómago. Celio Aureliano la atribuye a la debilidad del vientre; Themison la considera igualmente producida por la debilidad, llamándola reumatismo o incluso flato; Cornelio Celso da reglas para su tratamiento denominándola imbecilidad y en otro libro la describe entre los varios vicios que puede padecer el estómago. Alejandro de Tralles toma como causa la imbecilidad de la facultad retentiva y los humores viscosos. Pablo de Egina dice que además de las crudezas del estómago, juegan un gran papel los espíritus animales.

Los médicos árabes, especialmente Avicena, refieren la enfermedad al bazo e hígado, siguiendo a los griegos en sus explicaciones humorales. Fernel la sitúa igualmente en las vísceras antedichas, por una acumulación desmedida de humor melancólico. Senert viene a decir lo mismo, concediendo gran importancia a los espíritus animales. Igual opinan Boherhaave y su comentador Van Swieten. Carlos Pisón opina que es una superabundancia y estancamiento de la serosidad; Pablo Zachias la cree debida a un excesivo enardecimiento de las vísceras digestivas. Stahl la atribuye a la congestión de sangre abundante y demasiado espesa. Sydenham, tan poco amigo de síntomas y explicaciones teóricas, la interpreta como un desorden de los espíritus animales que produce fenómenos nerviosos. Federico Hoffman la atribuye a las afecciones de las vísceras abdominales. Willis admite la causa en cualquiera de los órganos, pero haciendo intervenir principalmente a los espíritus animales, los vapores malignos y otras influencias. Juncker a la lentitud y dificultad de la circulación de la sangre en la vena porta y vísceras que comunican con este vaso. Higmoore la hace depender de la constitución viciada y debilitada del estómago. Cheyne de las obstrucciones de las glándulas del estómago, intestinos y demás órganos del vientre. Peset y Vidal pasa por alto otros muchos médicos hasta llegar a los más modernos. Cullen considera el estudio de la gastralgia en tres secciones pues segrega a la pirosis de la dispepsia y a ésta de la secreción gaseosa o neumatosis. Villermay pone el asiento de la gastralgia en las vísceras abdominales y especialmente en el estómago, 
opinando que está afectado su sistema nervioso o sus propiedades vitales. Llega ahora a la escuela llamada fisiológica, de la que es el más representativo Broussais, y recoge su opinión de que es una forma crónica de la gastritis. Roche propone para explicarla un aflujo nervioso demasiado rápido y muy intenso o abundante en los tejidos afectados. Barras, finalmente, la considera de naturaleza nerviosa, así como otras enfermedades del estómago. Peset se inclina por este último. Todos los síntomas hacen pensar en la naturaleza nerviosa de la gastralgia. «Tan absoluta falta de lesiones orgánicas obliga a admitirla entre las neurosis, es decir, enfermedades sin daño material y apreciable, lo que significa simplemente que se ignora su naturaleza orgánica...». ${ }^{2}$

Diagnóstico en general y diferencial. De la gastralgia se conocen bien sus padecimientos, por lo que su diagnóstico general se realiza con bastante seguridad. En la gastralgia como en todas las neurosis puede hacerse un diagnóstico por exclusión o $a$ posteriori, desechando cuantas enfermedades tengan lesión, pero este método es complicado. Es mejor el diagnóstico a priori.

Parece sencilla la diferenciación entre una gastralgia o la gastritis, es decir, entre las enfermedades del estómago de origen nervioso o inflamatorio. En la práctica no sucede así, pues son enfermedades muy semejantes. La escuela de Broussais se obstina en no diferenciarlas, no queriendo reconocer el origen nervioso de la gastralgia.

Otra enfermedad que ofrece dudas y presenta un difícil diagnóstico diferencial en su periodo incipiente, es el cáncer de estómago o su simple ulceración. Cuando el cáncer está avanzado es fácil de diagnosticar. La úlcera simple de estómago tiene también sus síntomas. Lo mismo ocurre con la neuralgia intercostal, pero en la gastralgia se desordenan las funciones digestivas, no suele haber dolor a la presión ni tampoco punzadas en el trayecto del nervio.

Pronóstico. Lo realiza teniendo en cuenta dos conceptos, su gravedad absoluta y la relativa, es decir, sus mayores o menores probabilidades de curación. Respecto a la gravedad absoluta, se puede decir que esta enfermedad tiene un pronóstico halagüeño. En sus repetidos ataques es capaz de afectar a los tejidos materialmente, o contribuir de forma indirecta a que se propicie una lesión orgánica que muda por completo la faz del pronóstico. Es preciso, por tanto, no descuidarla para evitar efectos posteriores desastrosos. Si la gastralgia se ha desarrollado bajo el impulso de una causa moral, de una pasión fuerte y concentrada va a ser difícil su curación. Pero el elemento principal del pronóstico será siempre el grado de enfermedad. Termina Peset y Vidal este apartado exponiendo el grado de gravedad de las diferentes formas de gastralgia. La hipocondríaca es la más remolona, le sigue la dispépsica que ataca a

2 PESET y VIDAL, J. B. (1869), Historia de las neurosis llamadas digestivas, en especial la gastralgia, Valencia, pp. 66-67. 
la nutrición y luego la dolorosa que rara vez y por circunstancias especiales puede contribuir al desarrollo de lesiones orgánicas.

Terapéutica y tratamiento. Se compone de indicaciones que deben deducirse del examen de la enfermedad y sus fenómenos constitutivos, y también de sus causas, las condiciones orgánicas del enfermo, sus hábitos, régimen de vida y cuantos sean idóneos para ilustrar sobre la naturaleza de la misma. En el tratamiento racional, presenta varias indicaciones, a saber: combatir la influencia de las causas, tratar la enfermedad según su naturaleza y disminuir la violencia de sus síntomas, sobre todo el dolor. Las dos primeras constituyen el método curativo racional, la última el paliativo.

Tratamiento racional higiénico. Los medios curativos son de dos tipos, por una parte higiénicos y por otra farmacéuticos. Sin rechazar estos últimos se prefieren los primeros, cuyo modo de obrar es lento pero seguro. Según Francisco Valles ${ }^{3}$ el mayor número de enfermedades se curan por el buen régimen sin medicinas. Por más confianza que se tenga en los medicamentos, la gastralgia resiste a todos ellos y desaparece a veces espontáneamente auxiliada de la naturaleza, únicamente por la higiene. Esto no prueba la ineficacia de los medicamentos, pero acredita la superioridad indisputable de la higiene.

La experiencia acredita diariamente estas variaciones y nos da ejemplos de constituciones, que han cambiado por completo al modificar el método de vida. En definitiva, dice Peset: «Es el hombre un ser sumamente modificable y no pueden menos de inducir una variación particular a su organismo los agentes físicos y químicos que ejercen sobre él de continuo una acción constante». ${ }^{4}$ Cierta desconfianza muestra hacia los fármacos, ya que influye más sobre las neurosis gástricas el régimen que comprende los alimentos y bebidas, por la acción directa que produce sobre el organismo. La dietética no se reduce únicamente a los alimentos, sino que también examina los objetos que rodean al enfermo, haciéndoles cooperar a su restablecimiento. Peset y Vidal cree que la higiene y la dieta, con sus medios suaves y sencillos, son bastante eficaces para lograr por sí solas la curación de la mayor parte de las gastralgias.

Los medios morales han de tener influjo en unas afecciones que, como se ha dicho, están ocasionadas a menudo por las emociones del alma y otros desarreglos de la inervación. El médico debe por tanto buscar si alguna causa de esta naturaleza es origen de la gastralgia, que seguirá refractaria a cuantas medicaciones se utilicen, si no se modificara. Las frecuentes distracciones, el trato social, los ejercicios variados $\mathrm{y}$, sobre todo, los consejos de personas sabias e interesadas que gocen de algún prestigio sobre el doliente, podrán producir una curación más duradera. Hufeland propone tres cosas para curar a estos pacientes: valor, paciencia y prudencia. Ya que la enfermedad no es cosa de un día, el deber del médico es captarse la confianza del

3 LÓPEZ PIÑERO, J. Ma'; CALERO, F. (1988). Los temas polémicos de la medicina renacentista: las Controversias 1556, de Francisco Valles, C.S.I.C., Madrid.

4 PeSeT y Vidal, J. B., (1869), p. 88. 
sujeto, no vacilando en tomar cualquier determinación que juzgue conveniente, obrando con resolución y valentía.

Tratamiento racional farmacéutico. Hasta ahora se han expuesto los principales agentes higiénicos útiles y necesarios. Otros los suministra la farmacia, «tónicos fijos», porque dan fuerza a los tejidos aumentando su tenacidad, y avivan las funciones asimilativas. Generalmente, están indicados fuera de los ataques. Entre ellos merece preferencia la quina, ${ }^{5}$ base de casi todos los estomacales conocidos y con la que se consiguen los mejores resultados. Pero a la larga produce dolores de estómago.

Tratamiento racional de las diferentes formas. Hay tres formas de gastralgia, correspondiendo todas ellas a desórdenes en la inervación glanglionar. Aunque requieren las mismas pautas del tratamiento racional, presentan ciertas modificaciones, atendibles por la significación de su especial modo de representar el trastorno nervioso. La forma dolorosa, permite alimentos en mayor cantidad, admitiendo por lo tanto más por número de comidas. Son útiles los baños simples de agua tibia, que sirven de poderoso paliativo, sobre todo en caso de dolor violento. Se prescribirá una vida tranquila y quieta, exenta de cavilaciones o disgustos, evitando sobre todo los efectos bruscos de una pasión cualquiera, ya que las emociones vivas de todas clases son las que desarrollan el ataque. Para Peset es conveniente la administración de opiáceos, eficaces medios para calmar el dolor, precaviendo muchas veces su reaparición.

En la segunda forma, la dispéptica, convienen alimentos en corta cantidad, digeribles y nutritivos. Los baños se tomarán antes de la única comida, o de la más fuerte si se hicieran varias. Es indispensable evitar las pasiones de ánimo, especialmente las deprimentes.

Los medios farmacológicos para curarla deberán dirigirse a excitar y restablecer la función del estómago, o dirigir su estado de entorpecimiento y a regularizar y coordinar sus importantes funciones. La indicación principal consistirá en aumentar las fuerzas digestivas y disminuir la excitabilidad nerviosa general o local. Lo más oportuno son los excitantes, y los tónicos fijos y estomacales, a cuyo frente se halla la corteza de Perú o quina.

En cuanto a la forma hipocondríaca, se tendrá presente: alimentos de poco volumen, nutritivos, rechazando los flatulentos. Se les permitirá una o varias comidas según su costumbre, siendo conveniente que la cena se haga varias horas antes de acostarse.

$\mathrm{El}$ «tratamiento paliativo» trata de medicar el órgano ofendido, y corregir el desorden de sus funciones. En este caso el método empleado va únicamente dirigido contra el desarreglo funcional, contra los síntomas. Los agentes más útiles son los nervinos, calmantes y antiespasmódicos. Se asigna la palabra nervinos a los agentes

5 Sobre la introducción de la quina en Europa ver J. Ma . LÓPEZ PIÑERO; F. CALERO, (1992), «De pulvere febrifugo occidentalis indiae 1663», de Gaspar Caldera de Heredia y la introducción de la quina en Europa, Cuadernos valencianos de historia de la medicina y de la ciencia, Valencia. 
que aumentan directa y agradablemente la tonicidad del sistema nervioso, en parte o en su totalidad. Se utilizan los calmantes para el mayor desorden, otros son utilizados para su atonía o debilidad, denominándose excitantes de los nervios o neurosténicos, y obran aumentando el tono y las fuerzas de dicho sistema; los últimos son para su disminución y son llamados antiespasmódicos. También se asocian calmantes, que llevan el nombre de estupefacientes, los cuales modifican el sistema nervioso, suspendiendo o debilitando de un modo notable sus funciones. Los antiespasmódicos regularizan la acción deteriorada del sistema nervioso. Con los medicamentos que hasta ahora se han citado se consigue el tratamiento paliativo, o sea «la mitigación de los síntomas que llaman la atención por las mayores molestias que infieren; siendo los principales el dolor, que suele ceder a los de la primera sección o calmantes; la dispepsia a los de la segunda o tónicos excitantes, y el vómito y otras anomalías a los antiespasmódicos» 6 .

No es partidario de las sangrías y emisiones. Las emisiones sanguíneas aumentan el desorden de los nervios y tienden a eternizar las gastralgias. El otro método, el evacuante, comprende dos clases de medios distintos, eméticos y purgantes, según se promueven sus efectos por arriba o por abajo.

Los calmantes merecen con razón gran reputación, ya que es importante la desaparición de ataques, especialmente en la forma dolorosa. Su acción se reduce tan sólo a calmar los atroces padecimientos obscureciéndolos y a veces desvaneciéndolos circunstancialmente. De todas formas el opio y sus derivados son el remedio eficaz por excelencia. Otro método terapéutico para calmar los ataques de dolor es el baño tibio, pero ha de ser prolongado.

De todas formas, concluye Peset y Vidal, han sido muchos los remedios utilizados en las tres formas de gastralgia. Para él, estos medios farmacológicos, tan diversos, son absurdos, heterogéneos e inconcebibles. La mayor parte son resultado de las distintạs ideas sobre la naturaleza de la afección; otra parte, de un espíritu forzado de irritación y la menor tal vez de felices coincidencias.

Comprobación por la experiencia. El plan curativo de la gastralgia debe ser comprobado por la experiencia, de forma que vale más un hecho bien observado, que cien sistemas deslumbradores o cien explicaciones teóricas las más bellas y seductoras. Según Peset y Vidal para el progreso de las ciencias no hay más que un camino, el raciocinio fundado sobre la experiencia, ya que los raciocinios están sujetos a ser controvertidos cuando no son consecuencia inmediata de los hechos. «La medicina que obra con arreglo a las inspiraciones de la naturaleza, es la verdadera medicina, la única que descansa en las leyes eternas de la organización y la que desde Hipócrates acá ha sido siempre el bello ideal de los grandes médicos, de los prácticos célebres,

6 Peset y Vidal, J. B., (1869), p. 115. 


\section{MARIANO PESET MANCEBO}

resistiendo a todas las vicisitudes de los sistemas escolásticos» ${ }^{7}$. El estudio de la naturaleza, dice Peset, es la verdadera ciencia. Tiene nuestro autor recogidos varios casos de su práctica particular, que se pueden ver en mi tesis doctoral resumidos ${ }^{8}$.

\section{SOBRE EL CONCEPTO DE NEUROSIS}

Para la valoración de Peset y Vidal y las neurosis gástricas, comenzaré con un breve inciso sobre neurosis, apoyado en López Piñero y alguna lectura propia9.

La formulación de la neurosis de Pinel se concreta con la medicina anatomoclínica de la neurosis, ya que la interpreta como una alteración nerviosa sin hallazgo lesional. Pinel no da una definición específica, se limita a una mera descripción, distinguiendo entre neurosis propiamente dicha y las neurosis sintomáticas. Atribuye la primera a causas morales o simpáticas, mientras que las segundas dependerían de una lesión orgánica. Esta concepción incluye una idea de totalidad y de fisiologismo, que choca con un pensamiento como el anatomoclínico, ${ }^{10}$ localizador y anatómico. Después de Pinel, los anatomoclínicos intentan acabar con el concepto de neurosis como un resto inadecuado y envejecido. Frente a la propuesta de Cullen - enfermedad general- los anatomoclínicos no sólo hablarán de neurosis locales, sino que intentarán buscar su localización. Por ello esta afección aparece como un incómodo islote funcional en un conjunto de enfermedades perfectamente reducidas a términos de alteración anatómica localizada. Pero su concepto sigue siendo inabordable al conocimiento anatómico, fiel al planteamiento funcional del que había partido.

Otros autores, discípulos de Pinel, van a intentar una sistematización de la enfermedad. Así Anthelme Richeraud, desde la patología quirúrgica, aclara la clasificación de las enfermedades internas. Su sistema aparece en el momento en que la cirugía se está convirtiendo en patología quirúrgica, gracias al sostén teórico de la anatomía patológica y el modo de pensar anatomoclínico. Su nosología sistemática es una clasificación comprensiva de toda la patología, desde el punto de vista quirúrgico. Las ataxias son definidas como la aberración de las propiedades de la vida, y entre éstas se encuentran las enfermedades a las que los médicos dan el nombre co-

7 PESET y VIDAL, J. B., (1869), p. 129.

8 PESET MANCEBO, M. (1996), Juan Bautista Peset y Vidal (1821-1885) y la transición entre la medicina anatomoclínica a la de laboratorio, 3 t., tesis doctoral inédita, pp. 114-120.

9 Esta revisión del concepto de neurosis procede de la tesis doctoral de LÓPEZ PIÑERO, J. M $\mathbf{M}^{\mathrm{a}}$. (1963), Orígenes históricos del concepto de neurosis, Cuadernos valencianos de historia de la medicina, Valencia, pp. 90-129. PINEL, Ph. (1842), Compendio de nosología filosófica del Dr. Pinel, Madrid, p 351 y ss.; sobre cardialgia y gastrodinia, pp. 420 ss.

10 PESET REIG, J. L. (1993), Sobre el método anatomoclínico y las enfermedades mentales o vesanias véase pp. 167-183. Con el tiempo tendrán una explicación anatomopatológica, como irritación de meninges y otras lesiones. 
mún de neurosis. Da una caracterización que se corresponde, a grandes rasgos, con Cullen. Como neurosis se incluyen las neuralgias, convulsiones y las vesanias que no sólo comprenden la manía, melancolía y la demencia, sino también la hipocondría y el histerismo. En su obra se observa que el factor anatomoclínico es el responsable de la conversión de los antiguos «dolores» en «neuralgias».

Veamos ahora el concepto de neurosis en la patología anatomoclínica. Opinan que era un concepto anticuado y rechazable; la imagen de neurosis es lesionalmente negativa. Sin embargo, esta idea va a ir desapareciendo como consecuencia del descubrimiento de las lesiones que corresponden a las distintas enfermedades agrupadas bajo esta denominación. Unas enfermedades se incorporan a las restantes orgánicas, a medida que las lesiones anatómicas específicas sean descubiertas; otras se dilucidarán como puros síntomas o síndromes. Un número escaso de enfermedades no puede ser reducido a los esquemas lesionales anatómicos, y el concepto de neurosis en estos casos se utilizará de un modo provisional o simplemente impreciso e inadecuado ${ }^{11}$.

La nueva etapa de incorporación a la patología anatomoclínica del concepto de neurosis, sólo era posible tras una revisión a fondo que marcara sus límites y que enjuiciara todo el camino recorrido. Esta revisión la llevará a cabo E. J. Georget. El concepto de neurosis, depurado y rehabilitado de este modo, sólo va a encontrarse en aquellas patologías que sufrieron directamente su influencia. También reprochaba a Broussais el carácter especulativo de su doctrina. Georget acepta la labor de los anatomoclínicos, y de esta forma muchas enfermedades, antes consideradas como neurosis, quedan reducidas a enfermedades con lesión o a nuevos síntomas. Queda, por tanto, un pequeño reducto que sigue mereciendo este calificativo.

$\mathrm{La}$ aportación siguiente es un artículo de Foville, aparecido en 1834, en donde afirma que, bajo la denominación de neurosis, se han reunido confusamente todas las afecciones de causa material desconocida. Lo que intenta este autor es conseguir una caracterización positiva de la neurosis desde el punto de vista fisiopatológico. Ya que la investigación anatomopatológica no ha conseguido ningún avance procurará, con la investigación fisiopatológica, una localización funcional. En definitiva la fisiología ha sido llamada solamente para conseguir al menos una de las metas del método anatomoclínico: la localización. Más tarde Rosenthal, en su libro Tratado clínico de las enfermedades del sistema nervioso, sigue una pauta de localización anatómica estricta. Rosenthal pretende un encuadre de las diferentes neurosis en regiones anatómicas concretas. En definitiva incluye las neurosis dentro de un esquema anatómico, como las demás enfermedades nerviosas, pero al no conseguir una localización anatómica continúa con la hipótesis de una localización funcional. Con Charcot se llega a la culminación de la misma: en la neurosis existe una lesión ana-

11 Sobre el origen de las distintas nosografías de la época ver ARQuIOLA, E.; MONTIEL, L. (1993), La corona de las ciencias naturales. La medicina en el tránsito del siglo XVIII al XIX, C.S.I.C., Madrid, p. 244 ss. También sobre Pinel, como autor relevante de esta época, pp. 245-246. 
tómica localizada, pero es transitoria no siendo por tanto perceptible en un examen necróptico. A partir de aquí se considera la neurosis desde un punto de vista psicológico y antropológico ${ }^{12}$.

\section{SOBRE EL CONCEPTO DE NEUROSIS GÁSTRICA}

Para Peset y Vidal la gastralgia es una neurosis del estómago. La define como una neurosis de sensación y movimiento cuyo asiento es el sistema nervioso del estómago y cuyo carácter principal es carecer de toda lesión anatómica. No admite más gastralgia que la idiopática dividiéndola en las tres formas reseñadas.

Cercano a estas ideas de la gastralgia, Littré-Robin definen el término gastralgia en su Diccionario de Medicina y Cirugía (1889), como una «neurosis dolorosa del estómago, caracterizada por un sufrimiento vago en el epigastrio o más a menudo por un dolor vivo, lancinante con sensación de pinchazos, de desgarro, de calambre, de quemadura, etc.... ${ }^{13}$. Al final del trabajo hay una comparación entre Peset y Vidal y el concepto de gastralgia del diccionario médico de Littré-Robin. Aunque la diferencia en el tiempo es de veinte años, parece que este diccionario sigue a grandes rasgos la idea expresada por Peset y Vidal. Era la concepción de su tiempo; sin duda, se inspiraban en autores anteriores. Los dos consideran la gastralgia como una neurosis con dolores en epigastrio. En el apartado de anatomía patológica dice: «la ausencia de toda lesión en el estómago y demás vísceras del aparato digestivo forma el carácter fundamental de la enfermedad, según queda consignado en la descripción de las neurosis en general».

A continuación vamos a examinar los conceptos de neurosis y gastralgia en diferentes autores europeos de la época como Fabré, Monneret y Leven, para verificar lo que de semejanza o diferencia tiene Peset con estos médicos franceses. De este modo, precisaremos el nivel de conocimiento en el que se mueve el clínico valenciano. Para Leven las formas múltiples de la dispepsia, la gastralgia, la gastritis, etc., se confunden todas en una sola afección: la congestión patológica de la mucosa estomacal —una lesión anatomopatológica- determinada por la irritación del plexo solar. El sistema nervioso interviene en la producción de la dispepsia. Define la neurosis como «una enfermedad del sistema nervioso causada por la irritación de las

12 LITTRÉ-RoBIN, (1889), Diccionario de medicina y cirugía, 2 vols., p. 1009.

13 LiTTRÉ-RoBin, (1889); p. 1009. PESET y VIDAL, J. B. (1869), p. 23, su definición descriptiva como «neurose de sensación y movimiento, cuyo asiento es el sistema nervioso del estómago, y cuyos caracteres principales son carecer de toda lesión anatómica, desordenar del modo más variado y caprichoso las funciones que le están cometidas, ser apirética, no específicas, de marcha lenta, ordinariamente periódica ofreciendo ataques más o menos intensos y de poca gravedad». 
células nerviosas» ${ }^{14}$. La definición parece seguir el concepto de «irritación espinal» de los anatomoclínicos ingleses y, según López Piñero ${ }^{15}$, forma parte como neurosis particular de un campo colocado entre la histeria y las neuralgias. Para Leven será, en su primera etapa, una interpretación patogénica y a la vez fisiopatológica y de la realidad clínica de las neurosis. Cullen - sigue diciendo Leven- acuña la palabra neurosis para separar las enfermedades sin lesión de las enfermedades con lesión y pensó con ello proporcionar grandes luces a la ciencia médica. A juicio de Leven, en vez de hacerla progresar, detuvo su desarrollo. La anatomía patológica monopolizó la medicina y a pesar de los grandes servicios prestados a la ciencia perjudicó a la clínica, desde el día en que pretendió ser la base única del tratamiento de las enfermedades ${ }^{16}$. Según estas ideas parece que Leven critica el modo de obrar de los anatomoclínicos que supeditan la enfermedad a la lesión y descuidan lo que será una corriente posterior: la fisiopatológica.

Los patólogos, sirviéndose de la idea de Cullen, han creído que podían superar los fenómenos nerviosos que se refieren al estómago de los que pertenecen a la mucosa y han incorporado la gastralgia a la neurología; y las gastritis, al catarro gástrico; la úlcera, la dilatación, afecciones con lesión, han sido remitidas a otro grupo ${ }^{17}$.

De todas formas, Cullen atribuye a la anatomía patológica el conocimiento del órgano alterado en su estructura. En el capítulo dedicado a las enfermedades del estómago nombra los síntomas producidos por la denominada dispepsia como la hinchazón, espasmo, náuseas, vómitos, gases, etc. «He reunido en este capítulo - dice Levenlos síntomas del estómago y en el siguiente los del intestino grueso, porque la dispepsia, estudiada en la parte subdiafragmática del tubo digestivo, se desarrolla sucesivamente en estos órganos» ${ }^{18}$. Todos estos síntomas son provocados por la irritación del plexo solar. La función del estómago depende del estado fisiológico del plexo cuya irritación produce la dispepsia. Aunque la dispepsia se asemeja mucho a la gastralgia se puede decir que está en la misma línea de planteamiento de Peset y Vidal, aunque bastante posterior. Peset incorpora la dispepsia como una de las formas de la gastralgia.

Sigue comentando Leven en otro libro que cuantos la han abordado han emitido una teoría sobre ella. Interesa pues saber el estado actual de la ciencia e indicar lo que se ha dicho en los últimos años. Lasagne influido por Pidoux ha visto en la dis-

\footnotetext{
14 LEVEN, M. (1880), Tratado práctico de las enfermedades del estómago, Madrid, p. 5.

15 LÓPEZ PIÑERO, J.M ${ }^{\mathrm{a}}$. (1963), p. 177.

16 «La convicción general de que la experiencia clínica y anatomopatológica debía ser el punto de partida irrenunciable, llevó a muchos a suponer que la medicina jamás podría alcanzar la posición y el rigor logrado por otras ciencias...», pp. 244 de ARQUIOLA, E.; MONTIEL, L. (1993). Se ve en este pasaje cómo aunque los anatomoclínicos estaban entusiasmados por la localización de lesiones, no todos opinaban lo mismo.

17 LEVEN, M. (1880), pp. 7-8.

18 LEVEN, M. (1880), p. 199.
} 
pepsia una neurosis gástrica, es decir, una enfermedad sin lesión, un estado morboso enteramente igual a una neurosis cuyas manifestaciones son intermitentes. Por el contrario, esta neurosis del estómago no se separa nunca de dicho órgano y no se extiende nunca a los demás. Es un trastorno funcional y al sustituir una palabra por otra -neurosis gástrica por dispepsia- Pidoux y Lasagne no han hecho más que reproducir la idea de Barras ${ }^{19}$. Según Lasagne, en esta enfermedad se dan intermitencias así como en la bronquitis pero Leven opina que no las hay; sólo cuando haya falta de régimen o cualquier otra causa, se provocaría la dispepsia pero nada autoriza a ver en esta enfermedad intermitencias.

Tampoco ve motivo Leven para considerar como neurosis una dispepsia que reaparece en un individuo predispuesto, después de la menor fatiga, de la más insignificante emoción o de la transgresión más ligera del régimen higiénico, para desaparecer varios días después si se la somete a las exigencias de las normas adecuadas para recuperar la salud. Basta sólo la observación de un dispéptico durante años para decir que no se asemeja a una neurosis ${ }^{20}$. Leven en este libro no considera la dispepsia como una neurosis. Al asimilar - dice - la dispepsia a una neurosis, no se hace otra cosa, sino entrar en un terreno de pura especulación, colocándose fuera de la observación para entregarse a concordancias filosóficas que, por regla general, no son de ninguna utilidad. Más tarde cuando Lasagne intenta dar una definición de la dispepsia, lo hace pero con grandes dificultades. Según él, «dos condiciones se imponen para ser dispéptico: es preciso sufrir y quejarse» ${ }^{21}$. Ahora bien: hay dispépticos que no tienen sufrimientos ni verdaderos dolores y otros que tienen impresiones dolorosas y no se quejan. Es necesario - continúa - analizar las sensaciones penosas del estómago, pero Lasagne ha aclarado muy poco la cuestión de la naturaleza de la enfermedad cuando dice que no hay dispepsia sin dolor y sin quejas. Por su parte Barras quiere relacionar la dispepsia con enfermedades tan diferentes como la neurosị, herpetismo y artritismo. A esto opone Leven que la dispepsia es una entidad morbosa perfectamente delimitada. «La anatomía patológica ha obligado al clínico a no pagarse de palabras sino a buscar las lesiones anatómicas, la característica de las enfermedades». Puede verse en este párrafo la ya instaurada mentalidad anatomoclínica de Leven, así como en el siguiente en el que Leven utiliza técnicas microscópicas para apreciar lesiones gástricas, lo que le adscribe a una «medicina de laboratorio» que Peset y Vidal no vislumbra desde su postura de anatomoclínico puro, en estos momentos —quizá sí años después-.

19 LEVEN, M. (1889), Las neurosis, Madrid, p. 133.

20 LEVEN, M. (1889), p. 133. En este mismo libro se puede ver la opinión de Barras: «La gastritis desapareció, siendo reemplazada por la gastralgia, es decir, por una neuralgia del estómago sin lesión, ni congestión y ni irritación».

21 LEVEN, M. (1889), p. 149. 


\section{NEUROSIS Y GASTRALGIA SEGÚN JUAN BTA. PESET Y VIDAL (1869)}

El estómago es un órgano sujeto diariamente a inflamaciones. Las congestiones no se aprecian con claridad al hacer la autopsia. Sin embargo las lesiones glandulares, la degeneración de los vasos y las alteraciones del tejido celular son frecuentes y pueden estudiarse con el microscopio. En cuanto se examinó más de cerca el estómago, auxiliados de la experimentación fisiológica y un análisis microscópico detenido, dejó ver ese órgano singular que no sufría nunca lesiones no alterándose jamás a pesar de su continua función, hecho reconocido por Cullen. Antiguamente no se conocía más que una, el cáncer; pero ya en su época se diagnostica la úlcera. Revela, por tanto, unos conocimientos mas avanzados, sin duda, que los expuestos por Peset, que está en la medicina de su tiempo, bien que algo rezagado como corresponde a la España de entonces.

Fabré, otro autor al que hemos recurrido en este análisis comparativo, recoge el término neurosis, pero no menciona los términos dispepsia o gastralgia. Habla de neurosis como enfermedades o entidades nosológicas aisladas. Así define el esofagismo como una neurosis que constriñe el canal faríngeo-esofágico y que puede producir disfagia absoluta o impedir la deglución de cuerpos sólidos o líquidos. El hipo es considerado como una neurosis del estómago o de las vías respiratorias pero, como he dicho, lo considera como una entidad nosológica aislada. En otros tratados - Peset, Monneret o Leven - la bulimia es considerada como un síntoma de la gastralgia; por el contrario, Fabré lo considera como una neurosis aislada lo mismo que la hipocondría.

Veamos ahora lo que sobre este tema escribe Monneret en su Tratado elemental de Patología Interna ${ }^{22}$. Bajo el nombre de «neurosis del estómago»-dice- deben comprenderse los trastornos puramente dinámicos de los tres actos fisiológicos fundamentales que tienen lugar en esta víscera. Estos son: sensaciones, secreciones y movimiento. En último término se habla de la dispepsia o trastornos de la quimificación o digestión gástrica. De todas estas entidades nos interesa la gastralgia, nombre que según Monneret debe reservarse para la neurosis del estómago, caracterizada por un dolor variable en intensidad que tiene su asiento manifiesto en este órgano. El resultado final de estos actos morbosos complejos es la dificultad o imposibilidad de digerir, en definitiva la dispepsia. En el curso y pronóstico no se pueden considerar ni grados ni formas predominantes. Hasta aquí todo concuerda con los esquemas dados por Peset y Vidal, pero a continuación realiza Monneret una clasificación algo diferente a la de Peset. Así - dice nuestro autor- si absolutamente se pudiera distinguir en la gastralgia formas particulares, corresponderían a los trastornos siguientes: de la sensibilidad, de la secreción y de los movimientos propios del estómago. De esta forma tendríamos la siguiente clasificación: la gastralgia simple; la flatulenta; con calambre, náuseas y vómitos; cloroanémica e hipocondríaca. Respecto a la

22 MONNERET, M. ed., (1868), Tratado elemental de patología interna, 2 vols., Madrid. 
duración considera que es variable con tendencia a la cronicidad, durando desde 20 a 30 años hasta 1 año.

Por último veremos los términos neurosis, dispepsia y gastralgia en diccionarios médicos. En el Diccionario de Ciencias Médicas se dice de la palabra Dispepsia que la mayoría de los médicos adoptan la significación admitida por Galeno, denominándola bradipepsia, o lentitud en la digestión. Galeno entiende por dispepsia un vicio de la digestión que da lugar a la depravación de los alimentos en el estómago. «Con el nombre de neurosis se comprende un crecido número de afectos del sistema nervioso, muchos de ellos son muy poco conocidos, y no reconocen por causa ninguna alteración material orgánica» ${ }^{23}$. A continuación expone una clasificación y dentro de las Neurosis de la vida interior se encuentran las neurosis de la digestión. Entre ellas el espasmo esofágico, la dispepsia, bulimia y cardialgia. Este último término se asemeja en su definición al de gastralgia. Según Peset y Vidal será un sinónimo de gastralgia. Es un sentimiento de ansiedad y de opresión dolorosa en el epigastrio, con sensación de desfallecimiento.

El término Cardialgia en el citado Diccionario, y según Van Swieten, viene definido como un dolor acerbo y lento que se siente debajo del apéndice xifoides. Aunque la definición sí que parece corresponderse con la de gastralgia de Peset y Vidal, luego la clasificación es muy diferente, y no habla, en ningún caso, de su origen nervioso; de todas formas en la acepción neurosis sí que aparece la cardialgia como una neurosis de la digestión. La sintomatología y tratamiento se asemejan en algunas partes. Comparándola de un modo general con la definición dada por Peset y Vidal se parece poco, aunque el concepto sea el mismo. Distingue la cardialgia idiopática y la sintomática que se manifiesta en muchas de las calenturas esenciales, intermitentes, perniciosas, etc. Aún siendo un libro de 1825 coincide a grandes rasgos con Peset y Vidal.

En el diccionario de Littré-Robin, el término neurosis se define como. «Nombre genérico de las enfermedades que se suponen tienen su asiento en el sistema nervioso, y que consisten en un trastorno funcional sin lesión actualmente apreciable en la estructura de las partes, ni agente material apto para producirlas...».24 Aquí el término neurosis se asemeja al considerado actualmente. En cambio, define la gastralgia como una «Neurosis dolorosa del estómago, caracterizada por un sufrimiento vago en el epigastrio, o más a menudo, de desgarro, de calambre, de quemadura, etc, dolor que tiene su asiento al nivel del apéndice xifoides, se propaga a las hipocondrías y hasta la espalda.... ${ }^{25}$. El concepto de gastralgia vemos que se asemeja mucho a lo definido por Peset y Vidal. Además este diccionario admite que se puede encontrar

23 DICCIONARIO DE LAS CIENCIAS MÉDICAS, (1825), Madrid, p. 176.

24 LITTRÉ-RoBIN, E. (1889), Diccionario..., p. 462.

25 LITTRÉ-RoBin, E. (1889), p. 1009. 
una lesión en el estómago, por lo que la definición parece de planteamientos posteriores, no incluyéndolo dentro del concepto de neurosis.

Hemos examinado algunos autores médicos de la época para tratar de encuadrar las páginas de Peset. Desde luego, no es fácil la afirmación decidida de cuál es su nivel - conoce hasta Barras-, ni la determinación de los autores en que se apoyó o las observaciones propias. El tratado nos da una idea de sus lecturas, quizá no siempre directas aunque sí las más: desde Hipócrates hasta Cullen, Barras, etc. —puede verse en el cuadro de citas que incorporo al final. Hemos presentado algunos médicos que nos permiten saber que se movía al nivel de los tiempos y planteaba algunas cuestiones que estaban en su época y después - Leven, Monneret-. Por tanto, aunque pertenece al pensamiento anatomoclínico en su visión de la gastralgia, es capaz de exponer la enfermedad y sus propias observaciones.

Describe las tres formas de la gastralgia que conocemos: la dispéptica, la dolorosa y la hipocondríaca. En cuanto a la naturaleza de la enfermedad comenta que aún siendo la anatomía patológica la «antorcha de los movimientos modernos» ${ }^{26}$ no ha podido contribuir en lo más mínimo a la resolución del problema de la naturaleza de las gastralgias. No pueden conocerse las modificaciones vitales que constituyen la enfermedad. «La alteración del organismo que les da lugar - los síntomas - es inaccesible a nuestra investigación... ${ }^{27}$. La falta de lesiones orgánicas obliga a admitirla entre las neurosis, es decir, enfermedades sin daño material y apreciable, lo que supone que se ignora su naturaleza orgánica.

\section{UN ANÁLISIS DE CITAS}

Sin duda, su estudio sobre las gastralgias es uno de sus mejores escritos. El mismo Peset padeció esta enfermedad y anotó sus síntomas diariamente, siendo a la vez médico y enfermo ${ }^{28}$. Interesa examinar qué autores consultó.

26 PESET y VIDAL, J. B. (1869), p. 56

27 PESET Y VIDAL, J. B. (1869), p. 56.

28 Lo recuerda PESET CERVERA, V. (1905-1906), Terapéutica, materia médica y arte de recetar, 2 vols., Valencia, p. 512, y se encuentra en los manuscritos, algunos de estos anteriores, sobre todo en una estancia que hizo en el balneario de Panticosa. 
MARIANO PESET MANCEBO

\section{CITAS DE LA GASTRALGIA Y PORCENTAJES}

\begin{tabular}{|c|c|c|c|}
\hline AUTOR & Siglo & $\mathrm{N}^{\circ}$ Citas & PORCENTAJE (\%) \\
\hline Aecio de Amida & V-VI & 2 & 1 '1 \\
\hline Alejandro de Tralles & VI-VII & 6 & 3,3 \\
\hline Alsinet de Cortada, J. & XVIII & 1 & 0,55 \\
\hline Barbier de Amiens, J.B.G & XVIII-XIX & 1 & 0,55 \\
\hline Areteo de Capadocia & I & 2 & $1 ' 1$ \\
\hline Atheneo de Atalia & I & 1 & 0,55 \\
\hline Avicena & $\mathrm{XI}$ & 2 & 1 '1 \\
\hline Baglivi, G. & XVII & 1 & $0 ’ 55$ \\
\hline Barras, J.P.T. & XIX & 1 & $0 ’ 55$ \\
\hline Bichat, $X$. & XIX & 1 & $0 ’ 55$ \\
\hline Boherhaave, $\mathrm{H}$. & XVII-XVIII & 3 & $1 ' 65$ \\
\hline Bonnet, $\mathrm{Ch}$. & XVIII & 1 & 0,55 \\
\hline Broussais, F.J.V & XVIII-XIX & 1 & 0,55 \\
\hline Celio Aureliano & IV-V & 9 & 4'95 \\
\hline Celso & I a.C.-I & 12 & $6 ' 6$ \\
\hline Cheyne, G. & XVII-XVIII & 1 & 0,55 \\
\hline Crates el Tebano & V-IV a.C. & 1 & 0,55 \\
\hline Crichton, A. & XVIII-XIX & 1 & 0,55 \\
\hline Cullen, W. & XVIII & 4 & 2,2 \\
\hline Darwin, Ch. & XIX & 1 & 0,55 \\
\hline Demócrito & V-IV a.C. & 1 & 0,55 \\
\hline Diocles de Caristo & IV a.C. & 1 & $0 ’ 55$ \\
\hline Dubois, $\mathrm{P}$. & XIX & 1 & $0 ’ 55$ \\
\hline Ettmüller, M. & XVII & 1 & 0,55 \\
\hline Fernel, J. & XVI & 1 & 0,55 \\
\hline Fleury, L.S. & XIX & 2 & $1 ' 1$ \\
\hline Foderé, F. E. & XVIII & 1 & 0,55 \\
\hline Forest, D. van & XVI & 1 & $0 ’ 55$ \\
\hline Frank, J.P. & XVIII & 4 & $2 ’ 2$ \\
\hline Galeno & II-I a.C. & 7 & 3'85 \\
\hline Georget, E.J. & XIX & 1 & 0,55 \\
\hline González Centeno, V. & XVIII & 1 & 0,55 \\
\hline Good, J.M. & XVIII-XIX & 2 & $1 ' 1$ \\
\hline Higmoore, N. & XVII & 1 & 0,55 \\
\hline
\end{tabular}

126

Asclepio-Vol. L-1-1998 
NEUROSIS Y GASTRALGIA SEGÚN JUAN BTA. PESET Y VIDAL (1869)

\begin{tabular}{|l|c|c|c|}
\hline AuTOR & SIGLO & N $^{\circ}$ CITAS & PORCENTAJE (\%) \\
\hline Hipócrates & V a.C. & 21 & $11^{\prime} 55$ \\
\hline Hoffman, F. & XVIII & 8 & $4^{\prime} 4$ \\
\hline Home, F. & XVIII-XIX & 1 & $0^{\prime} 55$ \\
\hline Hufeland, Ch. W. & XVIII-XIX & 2 & $1^{\prime} 1$ \\
\hline Jobert & XIX & 1 & $0^{\prime} 55$ \\
\hline Juncker, J.J. & XVII-XVIII & 1 & $0^{\prime} 55$ \\
\hline Leon, A. de & XVI-XVII & 1 & $0^{\prime} 55$ \\
\hline Leuret, F. & XVIII-XIX & 1 & $0^{\prime} 55$ \\
\hline Linné, C. & XVIII & 3 & $1^{\prime} 65$ \\
\hline Littré, E & XIX & 1 & $0^{\prime} 55$ \\
\hline Lobera de Avila, L. & XVI & 1 & $0^{\prime} 55$ \\
\hline Mandeville, B. & XVII-XVIII & 1 & $0^{\prime} 55$ \\
\hline Marcial & II & 1 & $0^{\prime} 55$ \\
\hline Martinet, L. & XIX & 1 & $0^{\prime} 55$ \\
\hline Monneret, M. Ed. & XIX & 2 & $1^{\prime} 1$ \\
\hline Morgagni, G. B. & XVII-XVIII & 1 & $0^{\prime} 55$ \\
\hline Pablo de Egina & VI & 2 & $1^{\prime} 1$ \\
\hline Pinel, Ph. & XVIII-XIX & 1 & $0^{\prime} 55$ \\
\hline Piquer, A. & XVIII & 1 & $0^{\prime} 55$ \\
\hline Pison, C. & XVI-XVII & 1 & $0^{\prime} 55$ \\
\hline Platter, F. & XVI-XVII & 1 & $0^{\prime} 55$ \\
\hline Pomme, P. & XVIII-XIX & 1 & $0^{\prime} 55$ \\
\hline Portal, A. & XVIII-XIX & 1 & $0^{\prime} 55$ \\
\hline Priessnitz, V. & XIX & 1 & $0^{\prime} 55$ \\
\hline Sagar, J.B.M. & XVIII & 4 & $2^{\prime} 2$ \\
\hline Sauvages, F.B. & XVIII & 5 & $2^{\prime} 75$ \\
\hline Schmidtman, L. J. & XVIII-XIX & 4 & $2^{\prime} 2$ \\
\hline Sennert, D. & XVI-XVII & 1 & $0^{\prime} 55$ \\
\hline Sydenham, T. & XVII & 3 & $1^{\prime} 65$ \\
\hline Sorano de Efeso & II & 3 & $1^{\prime} 65$ \\
\hline Sorapan de Rieros, J. & XVI-XVII & 1 & $0^{\prime} 55$ \\
\hline Starck, K.W. & XIX & 1 & $0^{\prime} 55$ \\
\hline Stahl & XVII & 1 & $0^{\prime} 55$ \\
\hline Swediaur, F.X. & XVIII-XIX & 1 & $0^{\prime} 55$ \\
\hline Swieten, G. van & XVIII & 3 & $1^{\prime} 65$ \\
\hline Temisón de Laodicea & & 2 & $1^{\prime} 1$ \\
& & & \\
\hline
\end{tabular}


MARIANO PESET MANCEBO

\begin{tabular}{|c|c|c|c|}
\hline AUTOR & SIGLO & $\mathrm{N}^{\circ}$ Citas & PORCENTAJE (\%) \\
\hline Tesalo de Tralles & $\mathrm{I}$ & 1 & 0,55 \\
\hline Tissot, S.A. & XIX & 2 & $1 ' 1$ \\
\hline Trousseau, A. & XIX & 2 & 1'1 \\
\hline Valles, F. & XVI & 2 & 1'1 \\
\hline Vilanova, Arnau de & XIII & 1 & 0,55 \\
\hline Villermay, J.B.L. & XIX & 2 & $1 ' 1$ \\
\hline Vindiciano & IV & 1 & 0'55 \\
\hline Vogel, J. & XIX & 4 & $2 ’ 2$ \\
\hline Whytt, R. & XVIII & 1 & 0'55 \\
\hline Willis, Th. & XVII & 1 & 0,55 \\
\hline
\end{tabular}

Hay, sin duda, una fuerte presencia de la medicina griega: Hípócrates, Galeno, pero también Celio Aureliano y Celso, junto con otros epígonos griegos o alejandrinos: Alejandro de Tralles, Pablo de Egina, Aecio, etc. Son muy numerosos, representan el 40 por ciento de las citas. Ante tal cúmulo, que no se halla en otros autores de la época, hay tres explicaciones que no son exclusivas, sino complementarias:

1. En algún momento, según hemos visto, realiza una historia de la enfermedad, lo que justifica esa presencia, en parte, pero no toda. Aparecen también con un sentido de presente.

2. Y unida a la anterior, Peset es un historiador y, en todos sus escritos, hay una referencia a tiempos pasados. Es una especie de erudición u ornato en donde - como complemento- vierte sus lecturas constantes de clásicos y de autores de los que le separan muchos años. En su Topografía se aprecia la misma costumbre.

3. Pero, además, es un autor «intermedio», según la calificación que le dio hace años López Piñero. Significa que se halla situado en el quicio de las dos grandes etapas de la medicina. La historia médica se ha ido construyendo paulatinamente, en cada período hay avances, descubrimientos. Pero en el XIX se produce un cambio radical, que separa al pasado de los conocimientos médicos, desde Grecia hasta esta época. En patología, en fisiología, en terapéutica se ponen las bases de la medicina actual. A partir de este momento, queda abolida la medicina antigua: Hipócrates o Galeno, o, en buena parte, toda la medicina anterior. Pero Peset y Vidal vive en los años últimos y todavía cree en ellos, como pensamiento perenne, que los siglos han retocado, mejorado, pero no destruido. 
Con un eclecticismo que es capaz de absorber conocimientos de cualquier tiempo cita a los clásicos, a la vez que a otros autores de los siglos XVI a XVIII. Pero también conoce los autores del XIX, que han escrito sobre estas materias: Barras, Bichat, Darwin, Dubois, etc. Se puede calcular un porcentaje de 20 por ciento. Lo que no es demasiado, aun cuando las citas no dicen con exactitud - mejor el análisis que hemos realizado - sobre su actitud o aceptación de ideas más modernas.

En todo caso, comparado con otros recuentos de $\operatorname{citas}^{29}$, se muestra muy proclive a recoger textos antiguos. Lo cual nos habla de unas intensas lecturas, un deseo de aprovechar cuanto tiene a la mano, una erudición. En suma, un autor que considera la historia de la medicina como un instrumento más de la formación del médico. Una historia entonces, en buena parte viva, pues todavía no se había dado los pasos trascendentales de la nueva y actual medicina.

Su interés por los viejos autores está todavía vivo en su siglo. Todavía parece vigente la idea de Cabriada en el siglo XVII, original de Bernardo de Chartres, del siglo XI: «los modernos ven más por que están situados en los hombros de los gigantes, que son los viejos autores». Algunos médicos, como Avicena, Lobera, Vallés, Arnaldo de Vilanova o Sorapán de Riera, dan noticia de sus amplias lecturas - de su plantamiento histórico de la enfermedad como vimos-. Después aparece un fuerte núcleo de médicos del XVII y XVIII de la medicina ilustrada que tan bien conoce, pues hay un lapso de retraso, sin duda, en la medicina española. Insisto en que resulta más visible en un médico de amplia lectura y con marcado interés por la historia y la erudición —es posible que no sea tan aguda en otros coetáneos-. Baglivi, Bichat, Boerhaave, Cullen, los higienistas Foderé y Franck, Hoffmann, Hufeland, Morgagni, Pinel, Piquer, Sydenham, Sthal, Swediaur, Van Switen, Wyllis, entre otros, dan la pauta de sus ideas.

29 Por ejemplo Blasco y Jorro por FRESQUET FEBRER, J. L. (1988), «De la materia médica a la farmacología experimental». En: LOPEZ PIÑERO, J. $\mathrm{M}^{\mathrm{a}}$ et al., (1988), Las ciencias médicas básicas en la Valencia del siglo XIX, IVEI, Valencia, pp. 263-330. 